\title{
SURVEY OF LTE DOWNLINK SCHEDULERS ALGORITHMS IN OPEN ACCESS SIMULATION TOOLS NS-3 AND LTE-SIM
}

\author{
Ramprasad Subramanian, Pantha Ghosal, Shouman Barua, Shiqi Xing, Sinhlam \\ Cong, Haider Al Kim and Kumbesan Sandrasegaran
}

Centre for Real-time Information Networks,School of Computing and Communications, Faculty of Engineering and Information,Technology, University of Technology Sydney, Sydney, Australia

\begin{abstract}
The LTE/LTE-A has become a catchphrase for research and lot of research are being conducted and carried out in LTE in various issues by various people. New tools are developed and introduced in the market to interpret the results of the new algorithms proposed by various people. Some tools are open access which are free to use but some tools are produced by the companies which are not open access. In this paper some of the open access simulation tools like LTE-Sim and NS-3 are analyzed and LTE downlink scheduler algorithms are simulated using those tools. In LTE systems, the downlink scheduler is an important component for radio resource management; hence in the context of LTE simulation, a study between the downlink scheduler models between the simulators are performed.
\end{abstract}

\section{KEYWORDS}

Round robin (RR), Proportional fair (PF), Maximum throughput scheduler (MT), Throughput to average scheduler (TTA), Blind equal throughput scheduler (BET), Token bank fair queue scheduler (TBFQ), Priority set scheduler (PS).

\section{INTRODUCTION}

LTE evolved from the earlier 3GPP system known as Universal Mobile Telecommunication System (UMTS), which in turn was evolved from Global System for Mobile Communication (GSM). In 2004, 3GPP started the work on LTE. The main aim was to deliver high data rates with low latency. In the new architecture the circuit switched (CS) core was replaced by packet switched (PS) core, which takes care of voice and data traffic unlike its predecessor (UMTS) the voice functions was handled by CS core and data is handled by PS core. The main motive of the research in LTE is to deliver a peak data rate of $100 \mathrm{Mbps}$ in downlink $50 \mathrm{Mbps}$ in uplink. But the expectation of the data rates specified above was exceeded in the final system, which delivered the peak data rate of $300 \mathrm{Mbps}$ in downlink and 75 Mbps in the uplink. In LTE, the communication is available in different frequency bands, of different sizes. Furthermore, the LTE can use both the paired and un-paired bands for the communication. In paired, the same frequency is used in both the uplink and downlink but whereas in unpaired, the uplink and downlink uses different frequency bands. In LTE downlink transmissions, frame length of $10 \mathrm{~ms}$ are used and grouped by radio transmission. Then each radio frame is created by 10 subframes of DOI : 10.5121/ijwmn.2015.7201 
$1 \mathrm{~ms}$ duration. Therefore both the uplink and downlink uses ten subframes. Thereafter the subframes are divided into the two slots with the duration $0.5 \mathrm{~ms}$ for each part.

\section{SCHEDULING IN LTE}

The physical layer resources for uplink and downlink shared channel (UL-SCH and DL-SCH) are allocated by the evolved node (eNB). The resources that are allocated for the uplink shared channel and downlink shared channel contains of physical resources blocks (PRB) and modulation coding scheme (MCS). The bit rate is determined by MCS and the capacity is determined by PRBs. These allocation of MCS and PRBs are done for one or more TTIs and the duration for each interval for TTI is one subframe (1ms). The downlink control channel is carried by LTE PDCCH (physical downlink control channel). DCI has all the information about allocation of RBs, power control command, uplink grant and MCS to be used etc. The DCI messages are scheduled for every TTI for users to increases the control overhead during high traffic scenarios. The control overhead becomes a blockage in the case where the limited resources is used for control information and this may result in the degradation of quality of service (QoS). So in-order to maintain a middle path and to prevail over this problem an concept called persistent scheduling is introduced, in this scheme the idle and non-idle periods are preassigned by control overhead of the user specific RBs over a time sequence. The user know about the allocation of TTI/RB in advance and apart from that, the eNB is also aware of this preassignment in advance, when and where it should decode PDSCH (Physical Downlink Shared Channel), without any additional PDCCH overhead. Major disadvantage of the persistent scheduling is that due to various factors like user mobility, channel quality, doppler effect and interference etc the TTI/RB cannot be persistent in real time due. So in order to correctly decode at the receiver an average $\mathrm{RB}$ requires multiple transmission. For this reason, the research is going on in the semi persistent scheduling is ongoing.

In semi persistent signalling, the control signalling is greatly reduced and in this scheduling every allocation is not signalled and this will ultimately reduce the signalling load. For example in voice over IP (VoIP) application, if each frame in the downlink that has the duration for every $10 \mathrm{~ms}$ to $20 \mathrm{~ms}$ is signalled then this will greatly increase the overall signalling load and it will consume more bandwidth. Semi-persistent scheduling allows to setup an ongoing allocation that persists until it is changed. Both uplink and downlink can be provisioned with semi-persistent schedules.

\subsection{DOWNLINK SCHEDULING}

The cell radio network temporary (C-RNTI) information is obtained by the PDCCH, and this is a dynamic UE identifier and this is used as an indicator that indicates the impending downlink resource has been demultiplexed by the MAC and passed on to upper layers and is now scheduled for the UE. The recurrence of the semi-persistent scheduling is provisioned by the radio resource control (RRC). The usage of the scrambling codes denotes the scheduling schemes nature that is dynamic or semi-persistent for the C-RNTI on PDCCH. The PDCCH uses very low bandwidth because it will not carry lot of information unlike the downlink DL-SCH. 


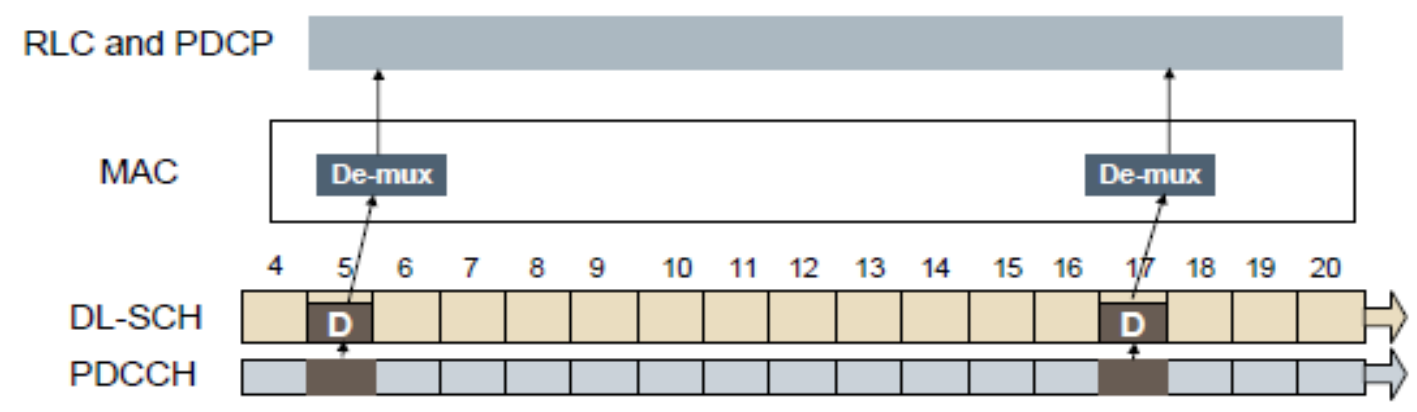

Figure. 1.Dynamic Scheduling (Freescale semiconductor et al.,)

The semi persistent scheduling information is included in Figure 2 from the Figure 1. Here, the semi persistence is provisioned by the RRC. This illustration shows a four-TTI example. When the first time it occurs there is signalling on the downlink PDCCH but after that, for every four TTIs transmission occurs without any signalling on the control channel. The dynamic scheduling is used for other purposes and this remains the same until it is changed by some other indication propagated in the control channel.



Figure. 2.Dynamic Scheduling (Freescale semiconductor et al.,)

\subsection{DOWNLINK SCHEDULING WITH HARQ}

Figure 3 is an incremental figure of the scheme specified in Figure 2. In Figure 2, the C-RNTI is carried by PDCCH and this adds to the downlink scheduling with HARQ. This indicates that the next downlink resource is scheduled for this UE. 
International Journal of Wireless \& Mobile Networks (IJWMN) Vol. 7, No. 2, April 2015

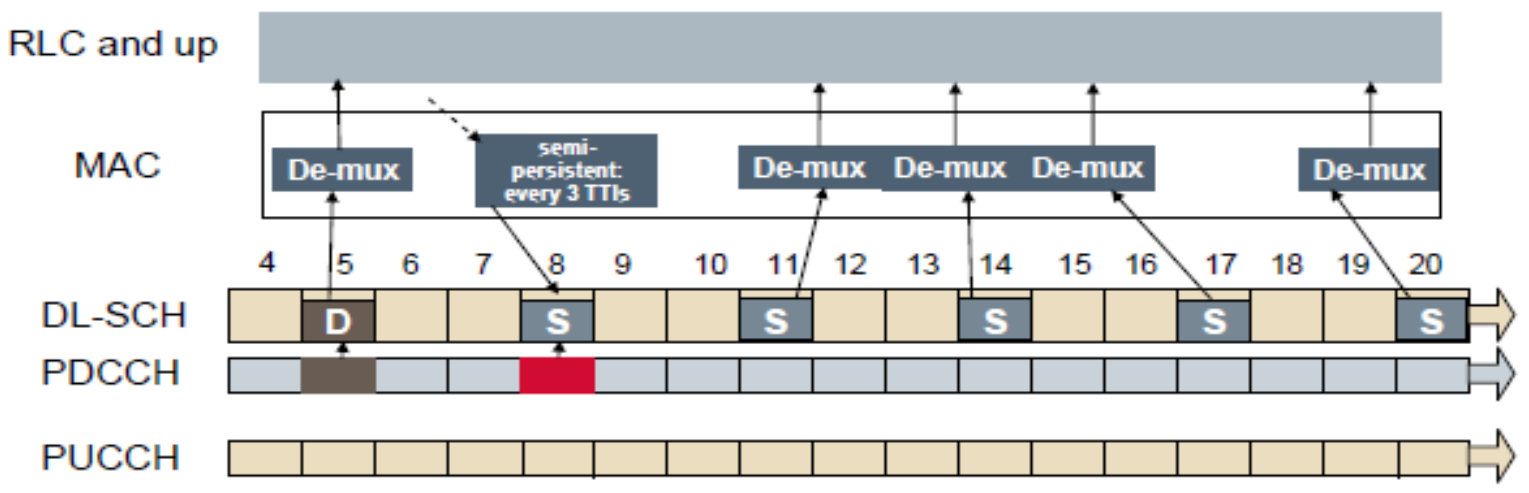

Figure. 3. Downlink Scheduling with HARQ(Freescale semiconductor et al.,)

In Figure 4 the ACK/NACK process that is specified is added to Figure 3 and HARQ process generates an ACK or NACK, which is sent on layer 1 or 2 (L1/L2) control channel (PUCCH) on the subframe $n+4$, on the every downlink transport block. Here in this situation if there is a negative acknowledgement, so a subframe needs to be transmitted again using HARQ. The retransmission is signalled dynamically and it is downlinked, then it is decoded and sent to upper layers. Again in the final, the subframe has to be again acknowledged. The process becomes intricate when both acknowledgements and semipersistent scheduling are involved.

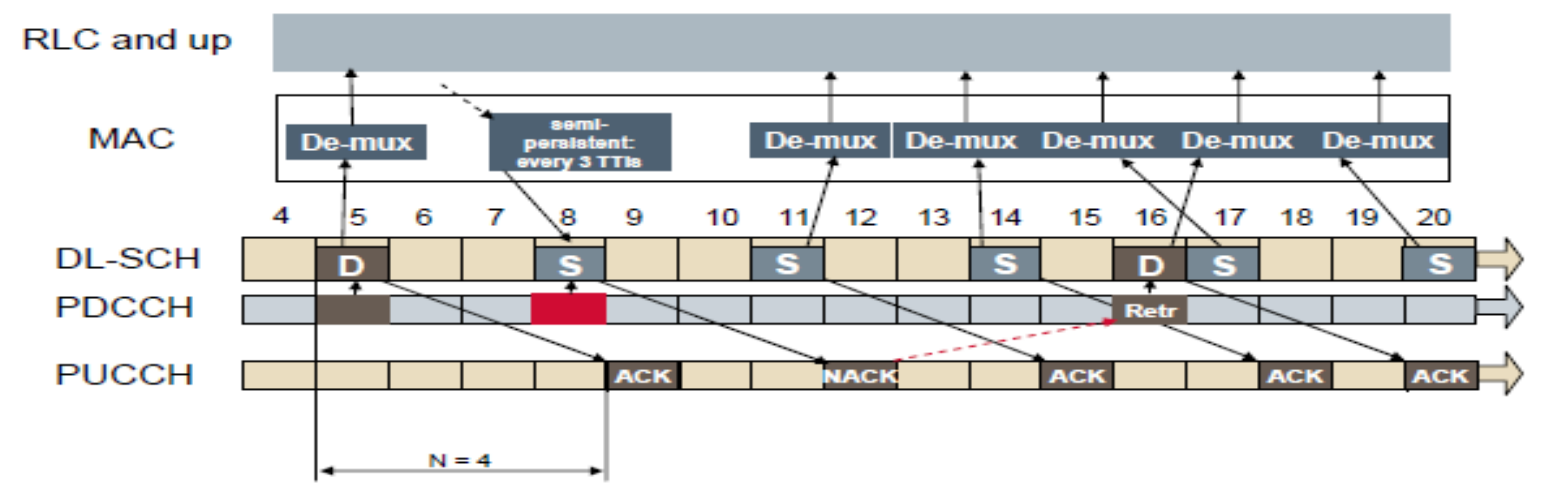

Figure. 4.ACK/NACK Process in Downlink Scheduling (Freescale semiconductor et al.,)

\section{FRAMEWORK}

Several LTE downlink algorithms have proposed by various researchers in academia and industry and in future several algorithms would be proposed and these algorithms would focus on several QoS aspects. So in order to provide a valid reference to the researchers in future who are working in this area an attempt is made to survey some of the models available in open access simulation tools like LTE-Sim and NS-3. In various forums there are lot of ongoing discussions about what simulation tools can be used for generating various downlink scheduling algorithms. So an attempt has been made to survey these two open access tools so that this paper will be a guide to future researchers. 
There are couple of algorithms NS-3 can support [1-2] and made available to the research community. Initially NS-3 came up with two scheduling algorithms namely Round robin (RR) and Proportional fair (PF). But more scheduling algorithms were needed for the research community. So in-order to make the NS-3 more powerful and useful to the research community, other algorithms like maximum throughput (MT), throughput to average (TTA), blind equal throughput (BET), token bank fair queue (TBFQ) and priority set (PSS) algorithms were added to the NS-3 repository[5]. These scheduling algorithms does the radio resource allocation either by time domain approach (TD) or frequency domain approach (FD). In the Time domain approach the LTE system assigns all the system resources to one UE during the particular transmission interval. In frequency domain the resource is allocated to UE based on frequency and time domains.

\section{NS-3 DOWNLINK SCHEDULING ALORITHMS}

\subsection{ROUND ROBIN (RR)}

This is one of the first algorithm that was supported by NS-3 and it is the simplest algorithm. This algorithm works like a ration where the time slots are assigned to every process in a equal share in the round robin fashion and in this algorithm all the process are handled without any priority. This algorithm is starvation free. The excess flows will be allocated in a circular fashion in another subframes, since it cannot be allocated in the same subframe. The MCS will be decided depending upon the received channel quality indicator (CQI) for each users.

\section{2 PROPORTIONAL FAIR (PF)}

In this algorithm, when the UE has high channel quality compared to the average channel condition with respect to the time, the resources are allocated to the UE[7]. with respect to the time. The PF can explained mathematically as:

Let users are denoted as $i, j$; let subframe index can be defined as $t$, and the resource blocks can be defined as $k$ be the resource block index; let modulation coding sequence (MCS) is defined as $M_{i, k}(t)$ for the user $i$ and on the resource block $k$ and let $S(M, B)_{\text {be the TB size in bits for }}$ that can serve and often it is the case where a number $B$ of resource blocks is used. The achievable rat that is represented as $R_{i}(k, t)$ in bit/s for can be specified as the below equation (1) user $i$ on resource block $k$ at subframe tis defined as

$$
R_{i}(k, t)=\frac{S\left(M_{i, k}(t), 1\right)}{\tau}
$$

where, the TTI duration of the $\tau$ can be the TTI duration. Each user is assigned with the resource block at the start of the every subframe $t$. In detail, the index $\widehat{i}_{k}(t)$ to which RB $k$ is assigned at time $t$ is determined as

$$
\widehat{i}_{k}(t)=\underset{j=1, \ldots, N}{\operatorname{argmax}}\left(\frac{R_{j}(k, t)}{T_{\mathrm{j}}(t)}\right)
$$


where $T_{j}(t)$ can be denoted as the past throughput performance professed by the user $j$. As per the above scheduling algorithm, a user can be assigned to various RBGs, that can be adjacent or non adjacent which depends on the present channel condition and the past performance on throughput $T_{j}(t)$. At the end of the subframe $t$ the past throughput performance can be determined by using the following exponential moving average approach:

$$
T_{j}(t)=\left(1-\frac{1}{\alpha}\right) T_{j}(t-1)+\frac{1}{\alpha} \widehat{T}_{j}(t)
$$

where $\alpha$ is represented as time constant (in number of subframes) of the exponential moving average, and the actual throughput can be denoted as $\widehat{T}_{j}(t)$ achieved by the user $i$ in the

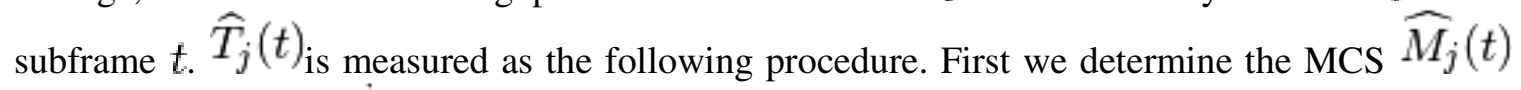
actually used by user $j$ :

$$
\widehat{M}_{j}(t)=\min _{k: \hat{i}_{k}(t)=j} M_{j, k}(t)
$$

then we determine the total number $\widehat{B}_{j}(t)$ of RBs allocated to user $j$ :

$$
\widehat{B}_{j}(t)=\left|\left\{k: \widehat{i}_{k}(t)=j\right\}\right|
$$

where $|\cdot|$ indicates the cardinality of the set; finally,

$$
\widehat{T}_{j}(t)=\frac{S\left(\widehat{M}_{j}(t), \widehat{B}_{j}(t)\right)}{\tau}
$$

\subsection{MAXIMUM THROUGHPUT SCHEDULER (MT)}

As the names suggests, the main idea of this algorithm is to provide the maximum throughput to a eNB [3][6]. In this algorithm, the RBG is assigned to each individual UE so as to attain maximum data rate in the current TTI.

Let the users can be denoted as $i, j$; and $t$ can be defined as the subframe index, and $k$ can be specified as the resource block index; let $M_{i, k}(t)$ can be defined as th MCS usable by user $i$ on the resource block and let $S(M, B)$ can be defined as the TB size in bits for the case where a number $B$ of resource blocks is used. The achievable rate can be defined as $R_{i}(k, t)$ in bit/s for the user $i$ on the resource block $k$ at the subframe $t$ can be defined as

$$
R_{i}(k, t)=\frac{S\left(M_{i, k}(t), 1\right)}{\tau}
$$


where $\tau$ can be specified as the TTI duration. At the beginning of the every subframe $t$, every user is assigned with the RB. In detail, the index $\widehat{i}_{k}(t)$ to which RB $k$ is assigned at time $t$ is determined as

$$
\widehat{i}_{k}(t)=\underset{j=1, \ldots, N}{\operatorname{argmax}}\left(R_{j}(k, t)\right)
$$

When in the case of multiple UEs with the same achievable rate, the present implementation always selects the first UE that is created in the script. Even though MT can provide maximum cell throughput but it cannot provide the fairness to UEs that are in experiencing poor channel condition.

\subsection{THROUGHPUT TO AVERAGE SCHEDULER (TTA)}

This scheduler algorithm can be assumed as an intermediate between MT and PF[3][6]. The metrics used in this algorithm can be calculated as follows:

$$
\widehat{i}_{k}(t)=\underset{j=1, \ldots, N}{\operatorname{argmax}}\left(\frac{R_{j}(k, t)}{R_{j}(t)}\right)
$$

Here, the achievable rate can be denoted as $R_{i}(k, t)$ in bit/s for user $i$ on resource block $k$ at subframe $t$ The calculation methodology is already discussed in the MT and PF. Meanwhile, $R_{i}(t)$ in bit/s denotes the achievable rate for $i^{\text {th }}$ user at subframe $t$. The difference in those two different achievable rates is how to get MCS. For $R_{i}(k, t)$, MCS can be calculated by sub-band CQI while $R_{i}(t)$ can be calculated by wideband CQI. TTA scheduler can only be implemented in frequency domain (FD) because the achievable rate of particular RBG is only related to FD scheduling.

\subsection{BLIND EQUAL THROUGHPUT SCHEDULER (BET)}

As the name suggest, this algorithm does not take the channel condition into consideration for resource allocation, BET provides equal resource to all the UEs in the same eNB[3][6]. Unlike Mt and TTA, this algorithm is a channel unaware scheduling algorithm. This algorithm uses wideband CQI in the scheduling decision in both frequency domain BET and time domain BET.

The scheduling decision by BET can be best explained by as follows:

$$
\widehat{i}_{k}(t)=\underset{j=1, \ldots, N}{\operatorname{argmax}}\left(\frac{1}{T_{\mathrm{j}}(t)}\right)
$$

where the past performance on throughput can be denoted as $T_{j}(t)$ by the user $j_{\text {and }}$ that can be calculated by the similar method as in PF scheduler. In the time domain flavour of the algorithm, the blind average throughput (TD-BET) scheduler selects the UE with highest priority metric and the it allocates all the RBGs to this UE. On the other hand, in the frequency domain flavour of the 
blind average throughput (FD-BET), every TTI, the scheduler selects the UE with the lowest average throughput in the past (largest priority metric). After this the scheduler, then assigns one RBG to this UE, it then calculates the expected throughput of this UE and uses this to compare with past average throughput $T_{j}(t)$ with other UEs. This UE will be assigned with the RBG by the scheduler until the expected throughput is better than the past throughput $T_{j}(t)_{\text {of all UE. }}$ Then the same method will be used by the scheduler to allocate RBG for a new UE which has the lowest past average throughput $T_{j}(t)$ and this happens until all RBGs are allocated to UEs. The principle idea behind this algorithm is that, in every TTI, the scheduler tries to achieve the equal throughput among all the UEs in the best possible way.

\subsection{TOKEN BANK FAIR QUEUE SCHEDULER (TBFQ)}

This algorithm is designed from leaky bucket mechanism and this TBFQ [4] is a downlink based QoS aware scheduler algorithm. The traffic flow of this scheduler is denoted as follows

$t_{i}:$ packet arrival rate (byte/sec)

$r_{i}$ : token generation rate (byte/sec)

$p_{i}$ : token pool size (byte)

$E_{i}$ : This is the counter that records the number of token borrowed from or given to the token bank by flow $i$.

$E_{i}$ can be smaller than zero.

Each $\mathrm{K}$ bytes of data consumes $\mathrm{k}$ number of tokens. Apart from this, this algorithm in order to balance the traffic between different flows, it maintains a shared token bank. If rate $r_{i}$ the token generation is bigger than rate $t_{i}$ the packet arrival, then the tokens overflowing from token pool are added to the token bank, and $E_{i}$ cab be increased by the same amount. Otherwise, the flow $i$ needs to withdraw the tokens from the token bank based on the priority metric and $E_{i}$ is decreased. Obviously, the user that contributes more for the token bank also has the highest priority to borrow the tokens; on the other hand, the vice versa also applies, that is the user who borrows more tokens from bank has the lowest priority to continue to withdraw tokens. Therefore, in the case of many users that are having the similar token generation rate, traffic rate and token pool size, suffers with higher interference and has more opportunity to borrow tokens from bank. Apart from this, this algorithm can also control the traffic by limiting the token generate rate to limit the throughput. Additionally, this algorithm can also maintain the following three parameters for each flow:

- $\quad$ Debt limit $d_{i}$ : if $E_{i}$ is less than certain threshold, user $i$ cannot borrow tokens further from the token bank. This mechanism is designed for preventing the malicious UE to borrow too many number of tokens.

- $\quad$ Credit limit $c_{i}$ : this can be defined as the maximum number of tokens that a UE $i$ can borrow from the token bank at one time.

- $\quad$ Credit threshold $C$ : This can be defined as, once when $E_{i}$ reaches the debt limit, UE $i$ can store $C$ tokens to the bank in order to further borrow tokens from the token bank. 


\subsection{PRIORITY SET SCHEDULER (PS)}

This is another QoS aware scheduler algorithms that combines both the frequency domain (TD) and time domain (FD) packet scheduling algorithms into one scheduler algorithm [3]. The fairness among the UE is controlled by defining a specified target bit rate (TBR). In the TD, the UE with non empty RLC buffer is selected by the scheduler and then they are divided into two sets based on the TBR:

set 1: UEs with the past throughput average is lesser than TBR; TD scheduler calculates its priority met- ric $p_{k}^{1}(\mathrm{t})$ following the BET.

$$
p_{k}^{1}(\mathrm{t})=\frac{1}{T j(t)}
$$

set 2: UEs with the past throughput average is larger (or equal) than TBR; TD scheduler calculates its priority metric $\mathrm{p} 2 \mathrm{k}(\mathrm{t})$ following the proportional fair $(\mathrm{PF})$ approach:

$$
p_{k}^{2}(\mathrm{t})=\frac{R j(k, t)}{T j(t)}
$$

Here, the achievable data rate $R_{j}(k, t)$ for the UE $j$ at time the $\mathrm{k}$-th RBG and $T_{j}(t)$ is the average past throughput of the UE $j$ and at the time $t$. The UEs that are belonging to the set 1 can be considered with a higher priority than the UEs in set 2 . This algorithm selects the Nmux UEs with the highest metric in the two sets and forward those UEs to FD scheduler.

\section{SIMULATION RESULTS OF VARIOUS SHCEDULING SCHEMES IN NS-3}

In the previous sections various scheduling algorithms has be defined theoretically and in this section we validate the scheduler and comparison would be made to validate the performance with some theoretical reference scenarios. The decision of validating against theoretical performance is mainly motivated by the lack of equivalent measurements from real LTE deployments that could be used for the same purpose. A particular set of scenarios are chosen with the simple assumptions so that it becomes possible to determine the theoretical performance of the scheduling and verify the accuracy of the implementations of the scheduler. We also note that, because of to these simplifying assumptions made, these scenarios might not be necessarily represent of real world conditions and deployments; in fact, the large scale simulation involving real time network conditions are beyond the scope of this paper, and are therefore these conditions are left for future study. For each well throughout scenarios, the reference throughput from each UE is calculated and this in turn cross verified with the obtained throughput to check whether this matches with the reference throughput within a given tolerance (equal to $10 \%$ of the throughput in this paper).

The simulation parameters values that are common to all the considered scenarios are given in the table 1. Since the phenomena of fading is not considered and the UEs are assumed to be stationary and it is configured as stationary nodes, because of this consideration each UE will have the same SINR for the entire whole simulation. In other words, both wideband and sub-band CQIs of UEs are assumed to be constants and their values are related to the distance between UE 
and eNB. The minimum allocation unit in the entire simulation is RBG and that contains two RBGs in the test cases. It is also stressed that the bitmap allocation is coded in allocation type 0 . In addition, we also use varying traffic patterns for QoS unaware and QoS aware schedulers. Specifically, the schedulers like MT, TTA and BET, are QoS unaware schedulers, it assumed that the RLC buffer for each UE is saturated always by using the ns-3 RLC saturation mode (RLC/SM) model. In this way, the simulation can fully reflect the RBG allocation behaviour of different scheduling algorithms. On the other hand, for the algorithms like TBFQ and PSS, the UDP traffics with different constant bit rates are generated in order to evaluate the unique feature of QoS aware schedulers.

All the schedulers that has been mentioned in this paper has been tested for two basic scenarios and they are:

- scenario I: In this scenario it is assumed that all the UEs are separated at the same distance to the eNB so that all the UEs can have same CQI both wideband and sub-band.

- scenario II: In this scenario, the UE are planned to have different distance to the eNB in order to have different CQI both wideband and sub-band.

The simulation time for all test cases for all the schedulers is 1 seconds in order to decrease the overall testing time in ns-3. This choice is acceptable due to the stationary UE and standard CQI throughout the simulation, which results in a very short union of time for the performance of the scheduling algorithms. In this section, we use TYX to indicate the UE reference throughput for any particular scheduler $\mathrm{X}$ and in scenario $\mathrm{Y}$. At the end of the each test result the number of UEs are represented as $N$. We would like to stress that the validation of the schedulers is mainly focused on the resources in the magnitude of throughput statistics for all the algorithms, based on the assigned have policies and resources in terms of bitrates and this work aims at verifying their correct design. The more realistic scenarios and conditions are left for future work and for future work apart from the scenarios we are also planning to take the conditions like delay, jitter, fairness etc will also be considered.

\begin{tabular}{|c|c|c|c|c|}
\hline $\begin{array}{c}\text { Distance between } \\
\text { the eNB and UE } \\
(\mathrm{Km})\end{array}$ & $\begin{array}{c}\text { UE throughput } \\
(\mathrm{KB} / \mathrm{s}) \\
\text { Reference } \\
\text { throughput }\end{array}$ & $\begin{array}{c}\text { UE } \\
\text { throughput } \\
(\mathrm{KB} / \mathrm{s}) \\
\text { FD-MT } \\
\text { throughput }\end{array}$ & $\begin{array}{c}\text { UE throughput } \\
(\mathrm{KB} / \mathrm{s}) \\
\text { TD-MT } \\
\text { throughput }\end{array}$ & $\begin{array}{c}\text { UE throughput } \\
\text { (KB/s) } \\
\text { TTA } \\
\text { throughput }\end{array}$ \\
\hline 0 & 2250 & 2250 & 2250 & 2250 \\
\hline 5 & 1750 & 1750 & 1750 & 1750 \\
\hline 10 & 800 & 800 & 800 & 800 \\
\hline 15 & 500 & 500 & 500 & 500 \\
\hline 20 & 200 & 200 & 200 & 200 \\
\hline
\end{tabular}


International Journal of Wireless \& Mobile Networks (IJWMN) Vol. 7, No. 2, April 2015

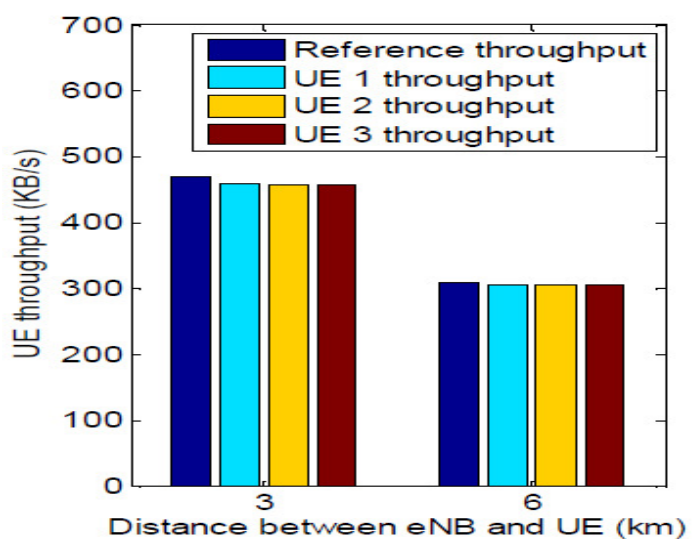

(a) FD-BET

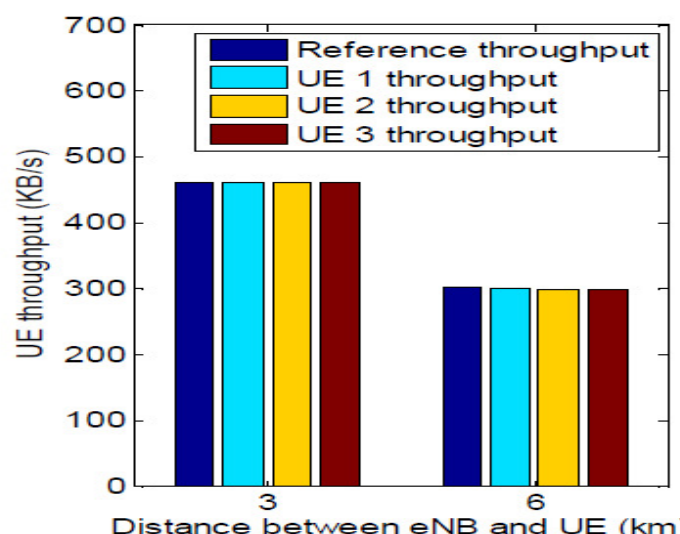

(b) TD-BET.

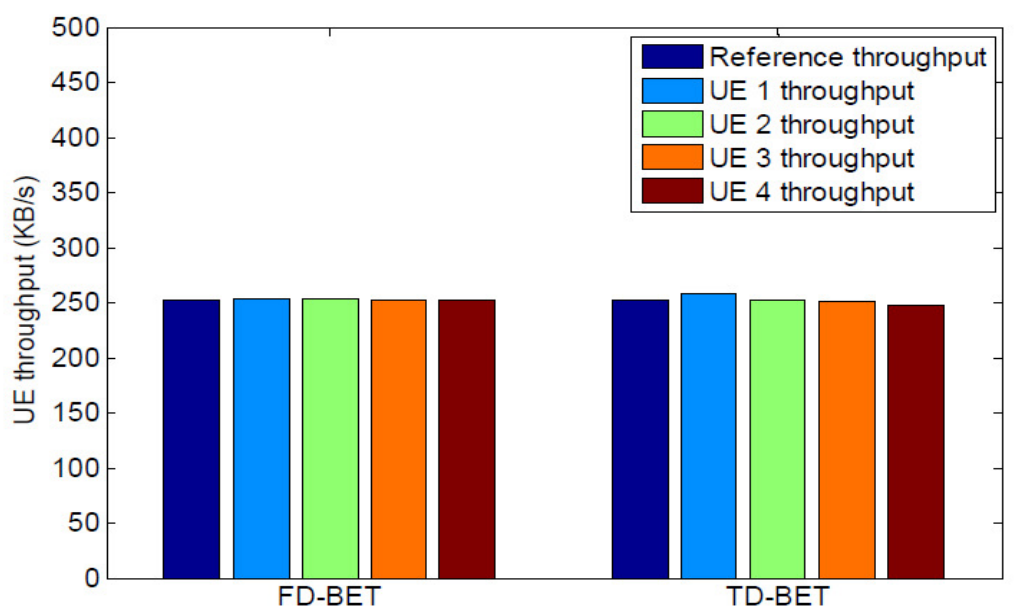

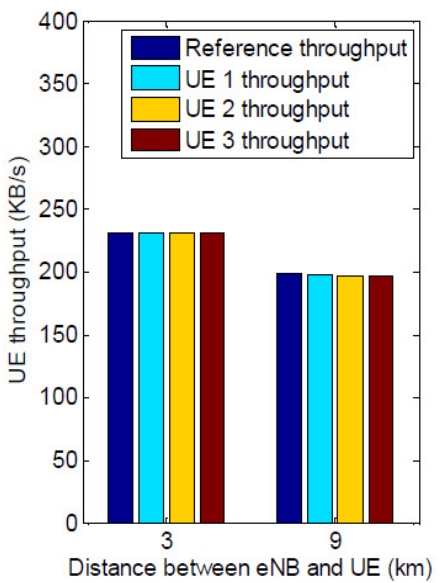

(a) FD-TBFQ.

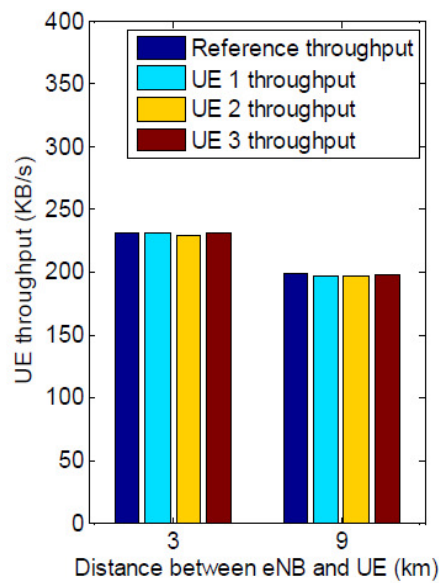

(b) PSS. 


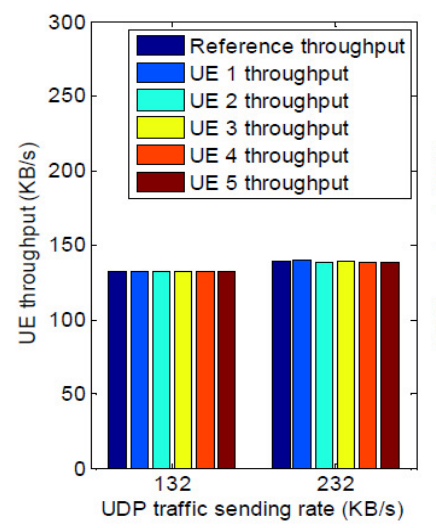

(a) FD-TBFQ.

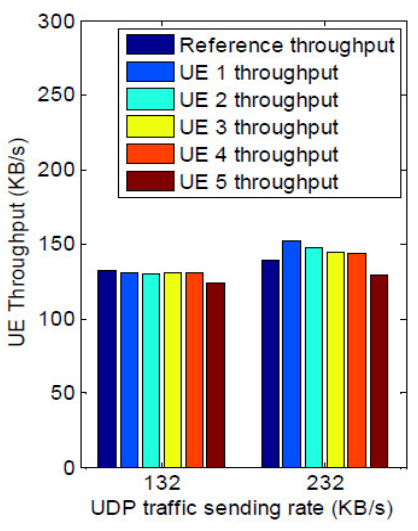

(b) PSS.

\section{LTE-Sim OPEN ACCESS SIMULATION TOOL FOR LTE}

To the best of the knowledge and unlike the other open source simulators, LTE-Sim has complete LTE protocol stack, multi-cell environments with uplink flows and realistic applications. LTESim is written in C++ and is a well-known object oriented plat and it is a event driven simulator. The four main modules of LTE-Sim as defined in [8] are: a) the Simulator, b) the Network Manager, c) the Flows manager and d) the Frame Manager. Now we will elaborate each functionalities: the simulator performs the function of starting - scheduling - running and stops every events, Frame Manager defines the LTE frame structure (starting and stopping of Subframes), the Network Manager creates each node and devices (eNB, Femtocell-Picocell, UE), manages positioning, Bandwidth and user mobility etc and the Flow Manager generates and handles applications such as VoIP, Video. Separate class is created for each network in the LTE architecture in this simulator i.e., eNB class, UE class, MME class etc. The whole of the LTE protocol stack is implemented with three network nodes, i.e., UE, eNB, and MME which forms the application to PHY layer that includes radio link control(RLC), radio resource control(RRC) and MAC entities. These network nodes can either be destination or source and maintain the flow of data through source and destination IP addresses, ports and transport protocol. The data flow, resource allocation and maintenance of QoS are performed by packet schedulers that are located at eNB. LTE-Sim supports some of the well known scheduling algorithms such as PF, MLWDF, ExPF, FLS and Log scheduling. LTE-Sim also supports CQI feedback by utilizing the channel quality estimation report from the UE and converting it to the set of CQI feedbacks reported to eNB and several other features. In the application layer of LTE-Sim, four traffic generators have been developed (trace-based, on-off, infinite buffer and constant bit rate). Packet transmission and propagation models are covered by the simulator's Channel module using four different phenomena as suggested in the path loss, the penetration loss, the shadowing and the fast fading due to the signal multipath. All the aforementioned features give it the flexibility and modularity to device a complete system for simulating LTE network in LTE-Sim.

\subsection{SIMULATION RESULTS IN LTE-SIM}

In this paper an evaluation of different packet scheduler algorithms such as PF, M-LWDF and EXP/PF supported by LTE-Sim is simulated in terms of packet loss Ratio, packet delay, average throughput, fairness index and Spectral efficiency with varying number of users and user 
speed. Simulation scenario is considered in an urban macro cell with cell coverage of $1 \mathrm{KM}$ with the presence on interference and propagation loss. Fairness index is measured by Jain's fairness [9] method. All the users are experiencing single flow (50\% of the users are having VoIP flow and the rest are having Video flow) modelled with infinite buffer application. Users (varied from 10 to 40 ) are moving with the speed either of $3 \mathrm{kmph}$ or $12 \mathrm{kmph}$.Simulation parameters is summarized in Table. 2

Table2. Simulation Parameters

\begin{tabular}{|l|l|}
\hline Bandwidth & $10 \mathrm{MHz}$ \\
\hline Frame Structure & FDD \\
\hline Cell Radious & $1 \mathrm{KM}$ \\
\hline User Speed & $3 \mathrm{Kmph}, 120 \mathrm{Kmph}$ \\
\hline Flow Duration & $80 \mathrm{sec}$ \\
\hline Maximum Delay & $.1 \mathrm{Sec}$ \\
\hline Video Bit rate & $242 \mathrm{kbps}$ \\
\hline
\end{tabular}

From Figure 9 it can be seen that for all the schedulers, average throughput (Video and VoIP) is decreasing with the increase in user speed. With the increase in user speed, the channel quality experienced by the user becomes worse and lower order MCS are selected which results in decreased average throughput of Video and VoIP flow.

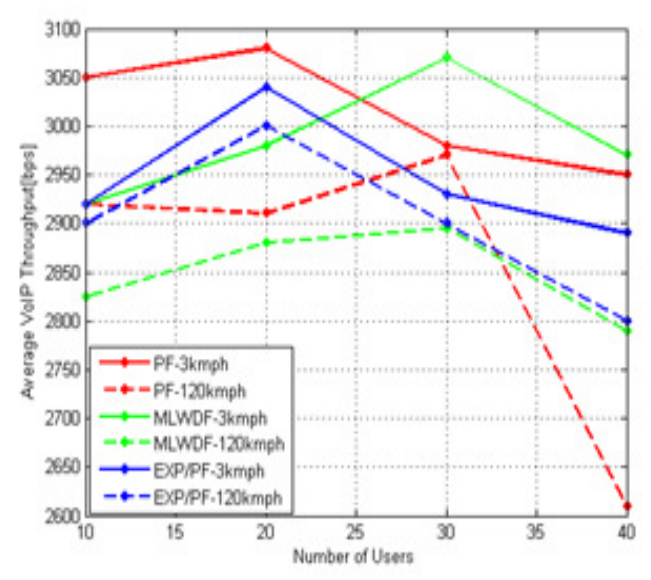

$9(\mathrm{a})$

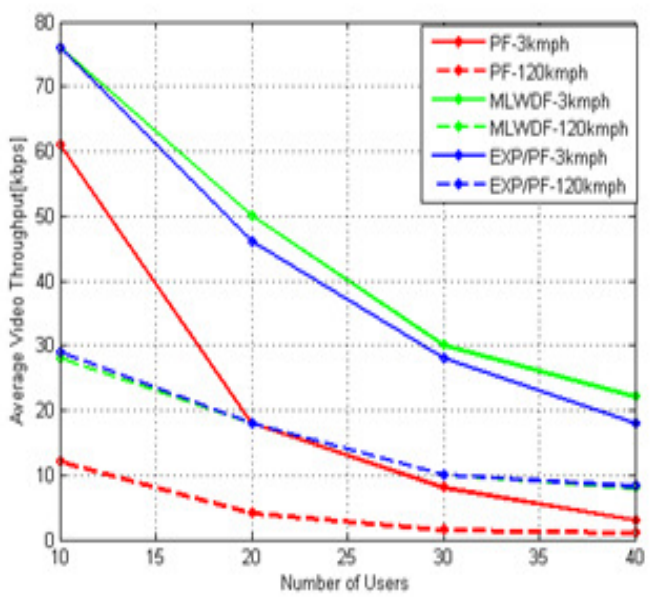

9(b)

Figure 9. Average Throughput of a)VoIP and b)Video Flow with different schedulers at different speed

Figure 10a shows, for video flow, packet delay remains almost the same for M-LWDF and EXP/PF with increasing user number and speed while delay increases with PF. For VoIP, packet delay increases with speed and user number for all the scheduling algorithms, which is show in Figure $10 b$. 


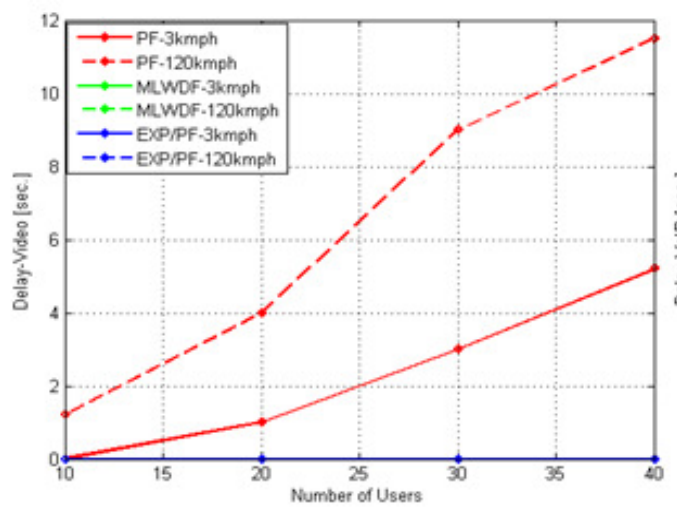

10 (a)

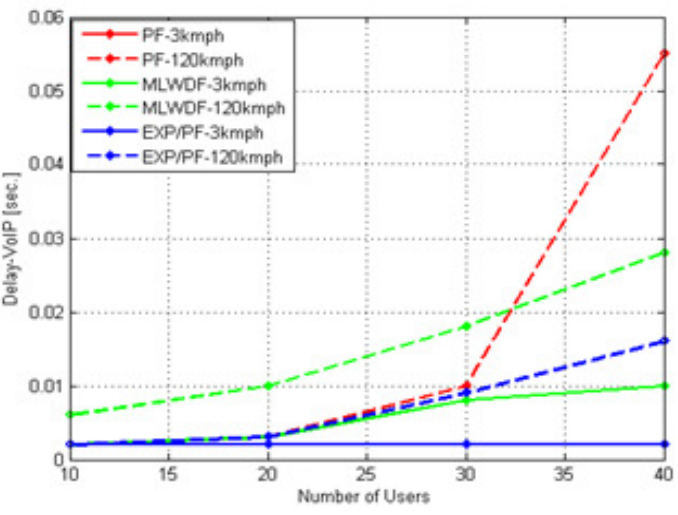

$10(b)$

Figure 10. Delay in a)Video and b)VoIP Flow with different schedulers at different speed

In LTE, link adaptation becomes complex with user speed which results in increasing packet loss, which is demonstrated in Figure.10, where for all the schedulers the packet loss increases with speed. Figure. 11 shows the spectral efficiency degrades with user speed irrespective of scheduling algorithms used

11(a)

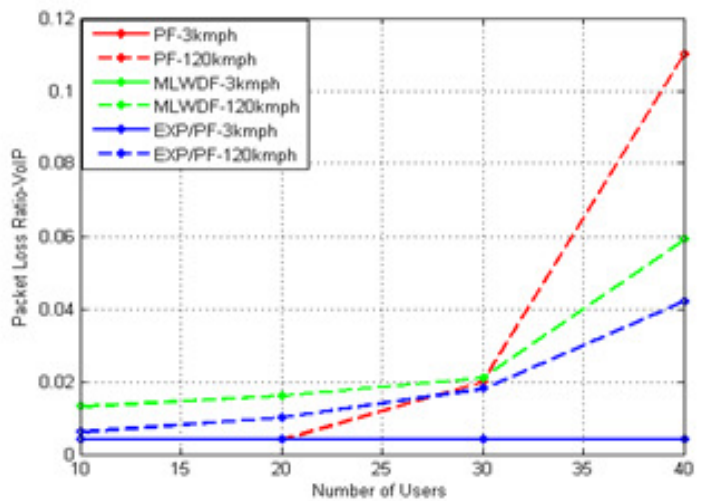

11(b)

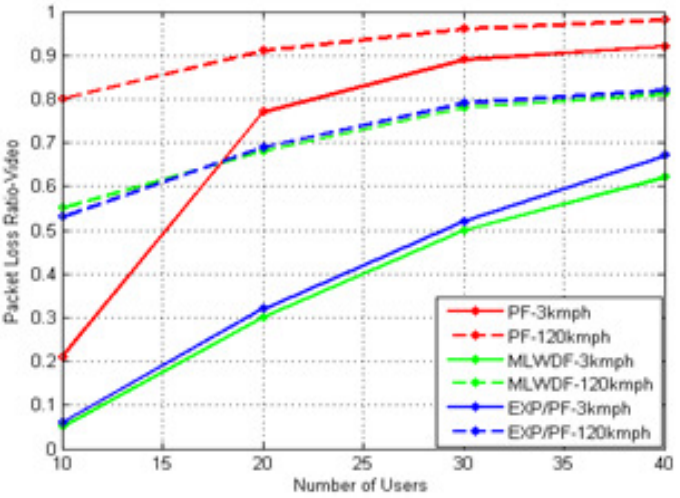

Figure 11. Packet Loss Ratio of a)VoIP and b)Video Flow with different schedulers at different speed 
International Journal of Wireless \& Mobile Networks (IJWMN) Vol. 7, No. 2, April 2015



Figure 12. Spectral Efficiency of different schedulers in LTE-SIM

\section{CONCLUSION}

NS-3 and LTE-Sim supports various downlink scheduler algorithms and the same has been simulated. Based on the area of work, traffic type such as voice or video, number of UEs, mobility type as speed, traffic environment such urban/suburban/rural the researchers can select the simulation platform either NS-3 or LTE-Sim to meet the needs. NS-3 provides a rich source of tutorials and source codes in the NS-3 repository site and since it is a open access tool these source codes can be used and modified as the simulation plan. LTE-Sim offers a vibrant users community in the Google group to raise any questions or doubts faced in the simulation. This special feature of LTE-Sim negates the lack of tutorials for the beginners. The uniform feature between these simulators are both work in Linux platform which is also another open source operating system. The install process for these simulators are pretty simple and can be used in the same computer. 


\section{REFERENCES}

[1] A. Ghosh, R. Ratasuk, B. Mondal, N. Mangalvedhe, and T. Thomas. LTE-Advanced: Next-generation wireless broadband technology. ACM Transactions on Multimedia Computing, Communications, and Applications, 17(3):10-22, Jun. 2010.

[2] Nicola Baldo, Marco Miozzo, Manuel Requena-Esteso, Jaume Nin-Guerrero, 'An Open Source Product-Oriented LTE Network Simulator based on ns-3', The 14th ACM International Conference on Modeling, Analysis and Simulation of Wireless and Mobile Systems, October 31-November 4, 2011.

[3] F. Capozzi, G. Piro, L. A. Grieco, G. Boggia, and P. Camarda. Downlink packet scheduling in LTE cellular networks: Key design issues and a survey. IEEE Communications Surveys \& Tutorials, 99(123), Jun. 2012.

[4] W.K. Wong, H.Y. Tang, and V.C.M. Leung. Token bank fair queuing: a new scheduling algorithm for wireless multimedia services. ACM Int. J. Commun. Syst., 17(6):519-614, Aug. 2004.

[5] Ns-3 lte scheduler repository. http://code.nsnam.org/dizhizhou/ns-3-dev.

[6] Femto Forum. LTE MAC scheduler interface specification, Dec. 2010.

[7] H. Seo and B. G. Lee, "A proportional-fair power allocation scheme for fair and efficient multiuser OFDM systems," in Proc. of IEEE GLOBECOM, Dallas, TX, USA, Dec. 2004.

[8] G Piro, L Grieco, G Boggia, F Capozzi, P Camarda,'Simulating LTE cellular systems: an opensource framework, " in Vehicular Technology, IEEE Trans. vol. 60, pp. 498-513, 2011.

[9] R. Jain, D. Chiu, and W. Hawe, A quantitative measure of fairness and discrimination for resource allocation in shared computer systems, Digital Equip. Corp., Littleton, MA, DEC Rep., DEC-TR301, Sep. 1984. 